\title{
PERFORMANCE OF THE SLOTLINE IN THE MM-WAVE RANGE
}

\author{
Ján Zehentner, Jan Macháč, Maurizio Migliozzi
}

Abstract - This paper deals with an investigation of the dispersion characteristics of the slotline in the mm-wave range. Attention is paid to the possible excitation of the surface leaky waves on the slotline. Higher order leaky waves were identified on the slotline made on a higher permittivity substrate. Their influence on the line characteristics is evaluated from the viewpoint of practice.

\section{INTRODUCTION}

The use of popular uniplanar circuits even within the mm-wave range has led to an advance in modern microwave and mm-wave technology. Both producers and users praise their desirable properties: low weight, small volume, operational reliability and simple production. The transmission line is the basic structural element of hybrid and monolithic circuits. The coplanar waveguide, coplanar strips and the slotline are typical representatives of uniplanar transmission lines. Considerable attention has been paid to investigation of the coplanar waveguide with infinite and finite outer strips [1], the slotline and conductor-backed coplanar strips [2], the covered conductor-backed coplanar waveguide [3], coupled slot lines [4] and microstrips [5,6]. Perhaps the most comprehensive study of the slotline can be found in [2]. Recently we have identified a new, so-called second leaky wave on the slotline, and the dependence of its dispersion characteristics on the slotline dimensions has been demostrated $[7,8]$. Mostly lossless lines have been investigated, sporadically also lines on the lossy substrate [9] and even lines with finite conductivity of the conductors [10] have been analysed.

Under specific conditions surface leaky waves may occur on the lines simultaneously with the bound wave, or they may be self-contained. Their excitation is undesirable since they increase line attenuation due to the transversal leakage of power. This results in cross-talk between neighbouring parts of the circuit. Leaky waves can also be excited at discontinuities, when they lower the quality factor of the respective circuit element. Consequently, it is useful to know the conditions for their excitation, and their behaviour dependent on frequency, line dimensions and material parameters.

Czech Technical University, Technická 2, 16627 Prague 6, Czech Republic 
In this paper we will present the results of an investigation of the slotline in a wide frequency range. After a short recapitulation of what is already known about the first leaky wave the existence of the second leaky wave on the slotline will be recalled. Its presence brings down the upper cut-off frequency of pure bound wave propagation. Closed-form formulae are available for easy calculation of this frequency. The second leaky wave asserts itself expressively on the slotline made on higher permittivity substrates when the characteristic impedance is app. 50-70 ohms. Detailed analysis of the slotline dispersion equation has revealed the possible occurrence of a third and a fourth leaky wave. These new findings enable us to evaluate the influence of the higher order leaky waves on the performance of the slotline in the mm-wave range.

\section{FIRST LEAKY WAVE AND OPERATION MODES}

A cross-section of the slotline is shown in Fig. 1. A laterally unlimited and lossless slotline is assumed. On a slotline with a relatively narrow slot the guided dominant mode is purely bound at frequencies below the so-called spectral gap. Within the spectral gap, solution of the dispersion equation is nonphysical either real or complex. Above the spectral gap the leaky mode can propagate, and its propagation constant is complex. The continuous increase of the slotwidth dramatically alters the dispersion curves, and for a sufficiently wide slot the bound mode and the leaky mode can propagate simultaneously. Consequently, the spectral gap now disappears. The frequency band of this simultaneous propagation may be fairly wide. The recently discovered new improper real solution of the dispersion equation serves to explain the continuous passing from the regime with a spectral gap to simultaneous propagation of the bound and the leaky wave due to the change in the slotline dimensions.

\section{DISPERSION CHARACTERISTICS OF THE SECOND LEAKY WAVE}

We have analyzed the open slotline by the method of moments modified as in the Galerkin testing procedure in the spectral domain with successive complex root searching. The propagation constant of the respective wave is a solution of the dispersion equation. The integration path in calculating its matrix elements was deformed from the real axis to include the residue contributions associated with propagation of the corresponding surface waves. We realized that - besides the solution of the first leaky wave associated with leakage into the $\mathrm{TM}_{0}$ surface wave - there can also be a solution belonging to the wave taking away power into the $\mathrm{TM}_{0}$ and $\mathrm{TE}_{1}$ surface waves simultaneously. We will call this field the second leaky wave. The 
dispersion characteristics of the second leaky wave are shown in Fig. 2 for the line taken over from [2]. It is seen that this wave can propagate at frequencies higher than $f_{2}$, at the point when the solution becomes physical. Evidently the former frequency gap from $f_{0}$ to $f_{1}$ now disappears and the bound wave can propagate simultaneously with the second leaky wave. The increase in $w / h$ causes the second leaky wave solution to become lower than $k_{0}$ and converts into a nonphysical solution. In the operation mode with the frequency gap, $\mathrm{f}_{0}$ determines the upper cut-off frequency of pure bound wave propagation. When the bound and the first leaky wave propagate simultaneously, $f_{1}$ gives this limit which is now lower than $f_{0}$. Similarly for the second leaky wave this frequency limit is identical with $f_{2}$. Closed-form formulae suitable for CAD have been proposed for these upper cut-off frequencies and hold when $0.01<\mathrm{w} / \mathrm{h}<1$ and $2<\varepsilon_{\mathrm{r}}<16$ [11]. The strong dependence of the second leaky wave dispersion characteristics on the line dimensions has been published in [8].

\section{THIRD AND FOURTH LEAKY WAVE}

It turns out that the first leaky wave asserts itself on a slotline with a wide slot, while the second leaky wave dominates on lines with a narrower slot manufactured on a higher permittivity substrate. For example, Fig. 3 shows that the bound wave and the second leaky wave can propagate simultaneously from $45.8 \mathrm{GHz}$ to $59.5 \mathrm{GHz}$, which cannot be neglected in the mm-wave range. Both the first and the second leaky waves may propagate at frequencies higher than $66.1 \mathrm{GHz}$. Additional examination of the solution of the dispersion equation resulted in the discovery of the third leaky wave, which takes away power into the $\mathrm{TM}_{0}, \mathrm{TE}_{1}, \mathrm{TM}_{2}$ surface wave simultaneously, Fig. 3. This wave may propagate at frequencies higher than $163.0 \mathrm{GHz}$. However, already at frequency $118.8 \mathrm{GHz}$ the physical solution belonging to the fourth leaky wave sets in. At the same time this wave leakes into the $\mathrm{TM}_{0}$, $\mathrm{TE}_{1}, \mathrm{TM}_{2}, \mathrm{TE}_{3}$ surface waves. Further solutions related to the third and fourth leaky wave which set in above the cut-off frequency of the $\mathrm{TM}_{2}$ and $\mathrm{TE}_{3}$ surface wave, are dropped in Fig. 3. In the case of the given example, the third and fourth leaky waves can be neglected. The dispersion characteristics of the first four leaky waves do not differ much when the permittivity grows from 10.8 to e.g. 12 and the remaining parameters are constant. The substrate thickness in Fig. 3 is $0.635 \mathrm{~mm}$ and in Fig. 4 it is $1.27 \mathrm{~mm}$, as in the standard sized substrate. However, the patterns change significantly with $w / h$. For comparison, Fig. 4 therefore shows them for $\mathrm{w} / \mathrm{h}=0.118$. All patterns are now shifted to lower frequencies lying in the mm-wave range, since $\mathrm{f}_{2}=24.64 \mathrm{GHz}, \mathrm{f}_{0}=27.54 \mathrm{GHz}, \mathrm{f}_{1}=29.36 \mathrm{GHz}, \mathrm{f}_{4}=65.81 \mathrm{GHz}, \mathrm{f}_{3}=80.98 \mathrm{GHz}$. 


\section{CONCLUSIONS}

Leaky waves can play an important role on the slotlines manufactured on higher permittivity substrates. Their occurrence falls in the mm-wave range and cannot be neglected. New third and fourth leaky waves have been revealed on the slotline. All leaky waves successively contribute to the transmitted field when the bound wave sets down and the frequency increases. Similar behaviour can also be expected on other printed-circuit lines. As an example, good agreement was found between our calculated and measured [12] propagation constant on the coplanar waveguide made on a silicon substrate with $\varepsilon_{\mathrm{r}}=11.67$ from 50 to $400 \mathrm{GHz}$.

\section{ACKNOWLEDGMENTS}

We gratefully acknowledge the scholarship granted by the Faculty of Electrical Engineering of the Czech Technical University in Prague to M. Migliozzi supporting his participation in this work. The work was done on the SP-2 computer at the Joint Supercomputer Center of the Czech Technical University, University of Chemical Technology and IBM Prague

\section{REFERENCES}

[1] M. Tsuji, H. Shigesawa, A. A. Oliner: New interesting leakage behaviour on coplanar waveguides of finite and infinite widths. IEEE Trans. Microwave Theory Techn., vol. MTT-39, Dec. 1991, pp. 2130-2137.

[2] H. Shigesawa, M. Tsuji, A. A. Oliner: Simultaneous propagation of bound and leaky dominant modes on printed-circuit lines: A new general effect. IEEE Trans. Microwave Theory Techn., vol. MTT-43, Dec. 1995 , pp. 3007-3019.

[3] Y. Lin, K. Cha, T. Itoh: Non-leaky coplanar (NLC) waveguides with conductor backing. IEEE Trans Microwave Theory Techn., vol. MTT-43, May 1995, pp. 1067-1072.

[4] Y.-D. Liu, Y.-B. Tsai: Surface wave leakage phenomena in coupled siot lines. IEEE Microwave and Guided Wave Lett., vol. 4, Oct. 1994, pp. 338-340.

[5] J. M. Grimm, D. P. Nyquist: Spectral analysis considerations relevant to radiation and leaky modes of open-boundary microstrip transmission line. IEEE Trans. Microwave Theory Techn., vol. MTT-41, Jan. 1993, pp. 150-153.

[6] D. Nghiem, J. T. Williams, D. R. Jackson: Existence of a leaky dominant mode on microstrip line with an isotropic substrate: Theory and measurement. 1993 IEEE MTT-S IMS Digest, Altlanta, GA, 1993, pp. 1291-1294.

[7] J. Zehentner. J. Macháč, M. Migliozzi: Upper cut-off frequency of the bound wave and new leaky wave on the slotline. 1997 IEEE MTT-S IMS Digest, Denver, CO, 1997, pp. 487-490.

[8] J. Zehentner, J. Macháč, M. Migliozzi: Evolution of new real and complex improper solutions of the slotine dispersion equation. 27th EuMC Conference Proceedings, Jenusalem, 1997, pp. 264-1269.

[9] H. Shigesawa, M. Tsuji: The nature of the spectral gap between bound and leaky solutions when dielectric loss is presented in printed-circuit lines. Radio Science, vol. 28, Nov.-Dec. 1993, pp. 1235-1243.

[10] T. Rozzi, F. Moglie, A. Morini, E. Marchionna, M. Politi: Hybrid modes, substrate leakage, and losses of slotline at millimeter-wave frequencies. IEEE Trans. Microwave Theory Techn., vol. MTT-38, Aug 1990, pp. 1069-1078.

[11] J. Zehentner, J. Macháč, M. Migliozzi: Upper cut-off frequency of the bound wave and new leaky wave on the slotline. Submitted to IEEE Trans. Microwave Theory Techn., 1997.

[12] W. Menzel, University of Ulm, private communication. 


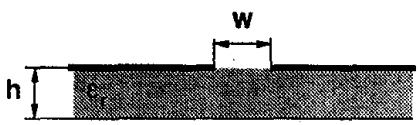

Fig. 1 Cross-section of the slotline

Fig. 2 The normalized phase $\left(\beta / \mathrm{k}_{0}\right)$ and leakage $\left(\alpha / \mathrm{k}_{0}\right)$ constants for the slotline with $w / h=0.4$ and $\varepsilon_{\mathrm{r}}=2.25$ as a function of normalized frequency $\left(h / \lambda_{0}\right)$
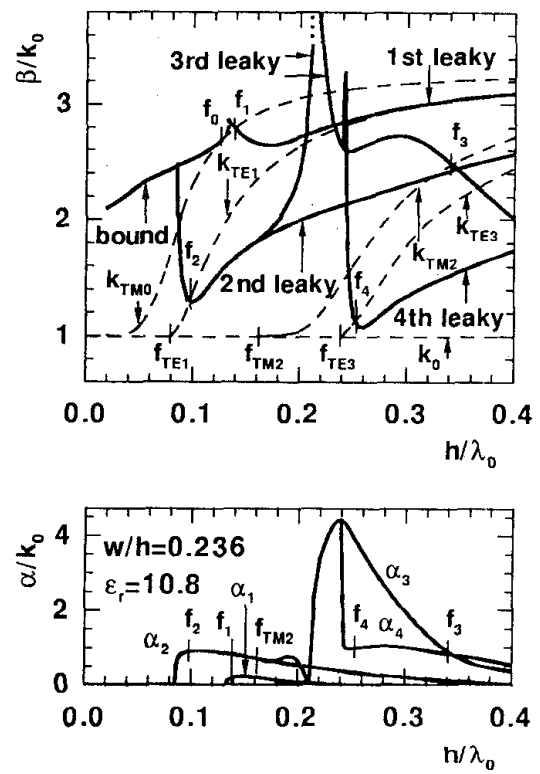

Fig. 3 The normalized phase and leaky constants of the first four leaky waves for the slotline with $\mathrm{w} / \mathrm{h}=$ 0.236 and $\varepsilon_{\mathrm{r}}=10.8$
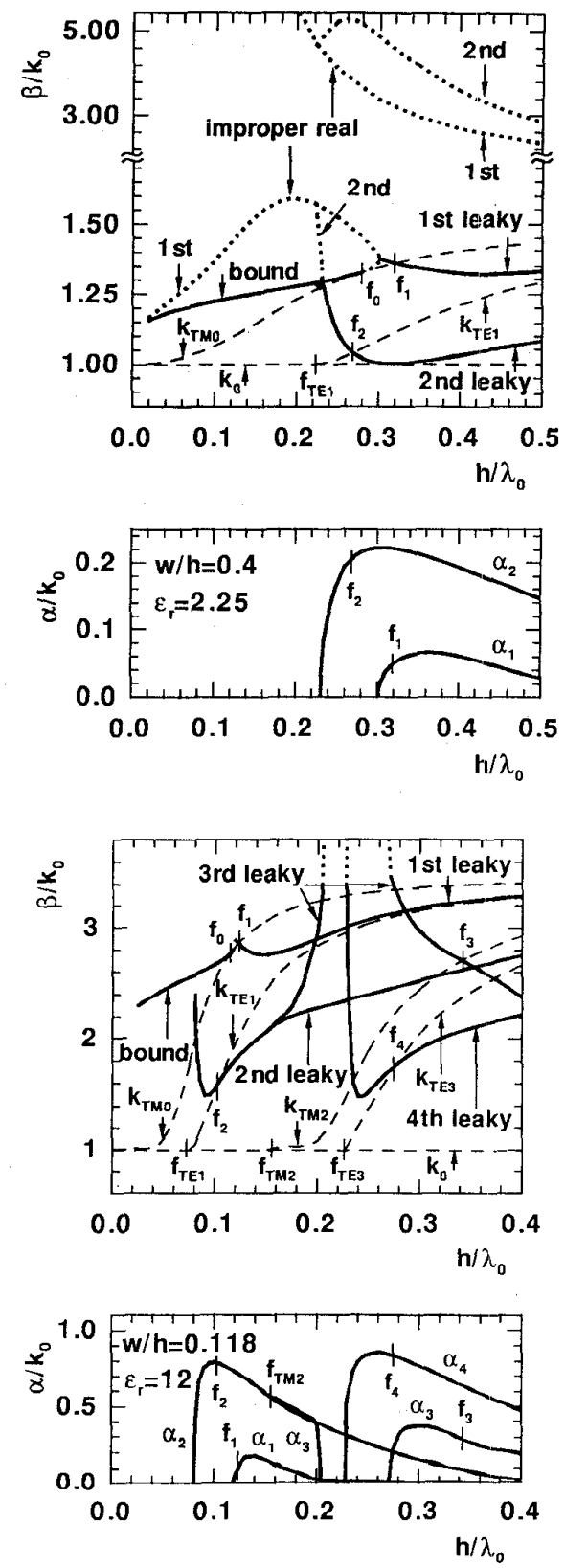

Fig. 4 A plot similar to that in Fig. 3 but now $\mathrm{w} / \mathrm{h}=0.118$ and $\varepsilon_{\mathrm{r}}=12$ 